\title{
Nonlinear plasma sheath potential in the ASDEX Upgrade 3-strap antenna: a parameter scan
}

\author{
W. Tierens ${ }^{1}$, J. Jacquot ${ }^{1}$, V. Bobkov ${ }^{1}$, J.M. Noterdaeme ${ }^{1,2}$, \\ L. Colas ${ }^{3}$, and the ASDEX Upgrade team ${ }^{1}$ \\ ${ }^{1}$ Max-Planck-Institut für Plasmaphysik, Boltzmannstrasse 2,D-85748 Garching, \\ Germany \\ ${ }^{2}$ Ghent University, Applied Physics Department, B-9000 Gent, Belgium \\ 3 CEA, IRFM, F-13108 Saint-Paul-Lez-Durance, France \\ E-mail: wtt@ipp.mpg.de
}

April 2017

\begin{abstract}
In this paper we use the SSWICH-SW software to calculate the (nonlinear) plasma potential near the ASDEX Upgrade 3-strap antenna for various operating parameters, and compare it with the (linear) parallel electric field strength and the (linear) RF potential. It is believed that the plasma potential is the cause of ion sputtering and additional heat loads on the antenna, and that the parallel electric field strength (as calculated by linear codes without sheath boundary conditions) is a good proxy for the plasma potential, and our results confirm the latter.
\end{abstract}




\section{Introduction}

In tokamaks, ICRF power is used to heat the plasma (among other heating methods). In the neighbourhood of the ICRF antenna, undesirable side effects are often observed, such as ion sputtering and additional heat loads [1]. This is likely due to the plasma sheath near the antenna, which accelerates the ions towards it.

Attempts have been made to avoid this problem by designing an antenna such that the sheath potential $V_{D C}$ is as low as possible [2]. On ASDEX Upgrade, a 3-strap 2-port antenna was designed such that the sheath potential becomes very small when the antenna feed scheme is chosen appropriately.

The 3-strap antenna was designed not by minimizing $V_{D C}$ directly (which involves nonlinear sheath physics), but rather by minimizing $E_{\|}[2]$, which can be calculated more easily using only linear physics. $E_{\|}$is thought to be the main cause of the sheath effects.

In this paper, we compare the results of linear calculations of the electric fields near the 3-strap antenna with the results of nonlinear $V_{D C}$ calculations. In sections 2 and 3 we discuss the SSWICH-SW and RAPLICASOL codes which we used to calculate this. In section 4 we show the results of our calculations.

\section{The SSWICH-SW code}

The SSWICH-SW code $[3,4,5,6]$ self-consistently determines the plasma sheath potential ("SSWICH" is short for "Self-consistent Sheaths and Waves for Ion Cyclotron Heating").

\subsection{Assumptions}

- SSWICH-SW considers only two frequencies: the antenna frequency $\omega$ and the zero frequency (DC).

- SSWICH-SW is $2 \mathrm{D}$, assuming negligible variation along the poloidal direction. The 3D information is reconstructed by running SSWICH-SW on consecutive 2D radial-parallel slices (figure 1).

- Simplified geometry: the background magnetic field is everywhere either parallel or perpendicular to metallic surfaces such as the antenna limiters.

- Slow wave only: $E_{\|}$is assumed to be entirely due to the slow wave. (hence the suffix "-SW" for Slow Wave. Recently, a Full-Wave version, SSWICHFW, has also been developed [7].)

- The perpendicular and parallel DC conductivity are assumed to be proportional to the parallel Spitzer conductivity. This is purely phenomenological, and the proportionality constants are not well constrained experimentally (see also section $5)$.
- No atomic physics in the sheath (no ionisation, recombination,...).

- Wide sheath approximation: we use SSWICH-SW in its asymptotic mode (see next subsection).

\subsection{Equations}

SSWICH-SW solves three coupled equations:

- The equation for the parallel electric field of the slow wave

$\left(\epsilon_{\|} \Delta_{\|}+\epsilon_{\perp} \Delta_{\perp}+\epsilon_{\|} \epsilon_{\perp} \frac{\omega_{0}}{c^{2}}\right) E_{\|}=0$

with boundary condition

$E_{\|}= \pm \frac{\epsilon_{s h}}{\epsilon_{\|} \delta} V_{R F}$

where $\delta$ is the sheath width.

- The radiofrequency potential $V_{R F}$ at the limiters in terms of $E_{\|}$

$\epsilon_{\perp} \Delta_{\perp} V_{R F}= \pm \epsilon_{\|} \partial_{\|} E_{\|}$

with boundary condition $V_{R F}=0$ at the outer edges of the simulation region and all boundaries parallel to $B_{0}$.

- The DC potential $V_{D C}$

$\left(\sigma_{\|} \Delta_{\|}+\sigma_{\perp} \Delta_{\perp}\right) V_{D C}=0$

(4) and (3) are coupled by the sheath boundary condition

$$
\begin{aligned}
J_{D C \|} & =-\sigma_{\|} \nabla_{\|} V_{D C} \\
& =i^{+}\left(1-\exp \left(\frac{V_{f}+V_{b}-V_{D C}}{T_{e}}\right)\right)
\end{aligned}
$$

where $i^{+}$is the local ion saturation current, $V_{f}$ is the floating potential (a constant, given the simulation parameters) and $V_{b}$ is the biasing voltage, a function of $V_{R F}$. In practice, (5) is limited by a saturation condition when the electron saturation current is reached. For further details see [3, 4]. SSWICH can be run in an iterative mode, where $E_{\|}$is calculated from $V_{R F}$ and $V_{R F}$ from $E_{\|}$until a self-consistent solution is found, but in this paper we will use its asymptotic mode where a wide-sheath approximation is used [5]: the sheath width $\delta$ is assumed to be large (which is equivalent to a high-power regime). $\delta$ then disappears from all equations, notably from (2), and the $E_{\|}$boundary condition becomes $E_{\|}=0$. In this approximation, $V_{R F}$ becomes a linear function of the port voltages. The asymptotic mode takes significantly less time to run than the iterative mode.

The DC potential is calculated in this way at the inner and outer sides of the vertical antenna limiters, which are indicated in red and blue in figure 1. Due to the slantedness of the SSWICH-SW coordinate system, data is exported beyond the vertical range of the antenna, as shown in figure 2. In further figures in this 
paper, we will omit data beyond the vertical range of the antenna, and use a common non-slanted coordinate system for the vertial coordinate (also shown in figure 2).

Also in figure 2, the green dashed lines indicate where SSWICH-SW imports an $E_{\|}$field map from another code (here RAPLICASOL, see the next section), to use as boundary condition in (1). The orange dashed lines indicate where SSWICH-SW uses $E_{\|}=0$ as boundary condition. On the gray dashed lines, no $E_{\|}$boundary condition is imposed. This boundary condition is the only mechanism which excites $E_{\|}$within SSWICH-SW. This is a limitation of SSWICH-SW: in reality, antenna limiters are not only passive elements which "receive" the slow or the fast wave. The limiters are also active elements of the antenna, and $E_{\|}$is affected by both the connections of the limiters to the RF power source (i.e. antenna design including limiters) and by propagation of the waves (including the fast wave) from other active elements to the limiters.

\section{The RAPLICASOL code}

The SSWICH-SW code takes as input an $E_{\|}$field map at the antenna aperture, which is calculated by a linear solver. Here we have used RAPLICASOL, a $3 \mathrm{D}$ frequency-domain finite-element solver which solves Maxwell's equations in the cold plasma approximation in the neighbourhood of a realistic albeit slightly simplified planar antenna geometry (figure 1).

The RAPLICASOL geometry includes two metallic bars above and below the antenna, which are also present in reality. All metallic elements, i.e. the antenna itself and these two bars, have perfectly conducting boundary conditions.

The RAPLICASOL simulation region is terminated by absorbing boundary conditions (so-called Perfectly Matched Layers or PMLs) on all sides except the outer wall, which is a perfect electrical conductor ("PEC") boundary condition.

For our simulations, we used the following parameters

- Parallel extent of the simulation region: $2.1 \mathrm{~m}$

- Vertical extent of the simulation region: $2.1 \mathrm{~m}$

- Radial extent of the simulation region (excluding PMLs): $20 \mathrm{~cm}$

\section{Results}

\subsection{Parameters}

All results in this section are obtained with the following parameters: the plasma is a pure deuterium plasma with 1D density profile obtained from ASDEX

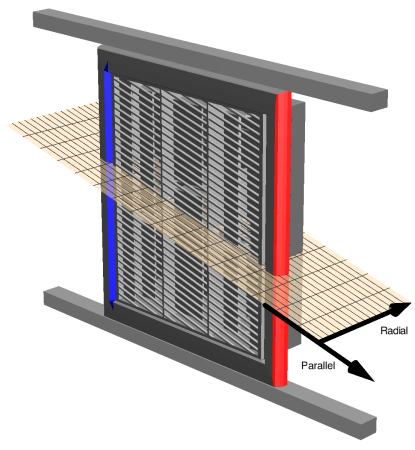

Figure 1. The RAPLICASOL model of the ASDEX Upgrade 3 -strap antenna. The slanted plane is a single 2D radial-parallel SSWICH-SW slice. The outer side of limiter 1 is shown in red. The inner side of limiter 2 is shown in blue.

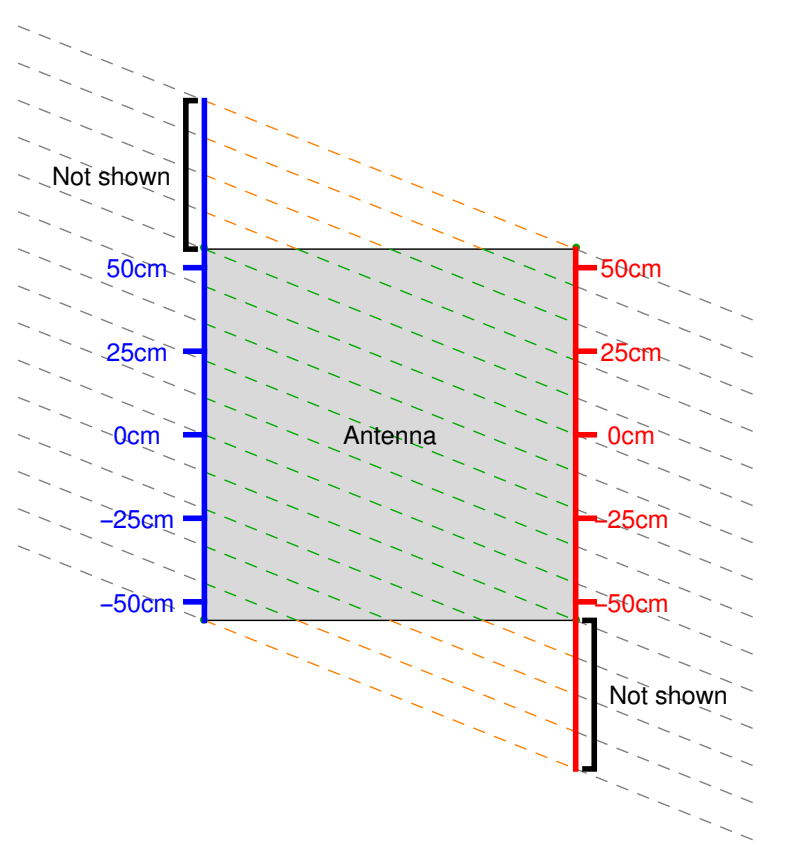

Figure 2. SSWICH-SW slanted coordinate system. The rectangle is the antenna aperture. The dashed lines are lines of constant "vertical position" in the slanted SSWICH-SW coordinate system. The red and blue lines are the poloidal locations of the left and right limiters, matching figure 1. On both limiters, SSWICH-SW exports data beyond the vertical range of the antenna, which will be omitted in other figures in this paper (regions indicated with "not shown" in the figure). In other figures in this paper, we use the non-slanted vertical coordinate system shown in red and blue.

Upgrade experiment \#31278 (figure 3). RAPLICASOL and SSWICH-SW can handle 3D density profiles, but we have not made use of this in this paper. The antenna frequency is $30 \cdot 10^{6} \mathrm{~Hz}$, and the electron temperature $T_{e}$ is $10 \mathrm{eV}$, and constant throughout the simulation region. The power balance is calculated assuming a total power of $1 \mathrm{MW}$.

The version of the SSWICH-SW code we use is version 10. The (dimensionless) transport parameters $R_{s, f p}$ and $R_{s, p p}$, which SSWICH-SW uses to model 


\begin{tabular}{|l|c|c|c|}
\hline SOL & $\max \Delta$ & radial scaling & parallel scaling \\
\hline Private & $1 \mathrm{~cm}$ & 100 & 4 \\
Free & $1 \mathrm{~cm}$ & 50 & 1 \\
\hline
\end{tabular}

Table 1. SSWICH-SW meshing parameters (spatial resolution)

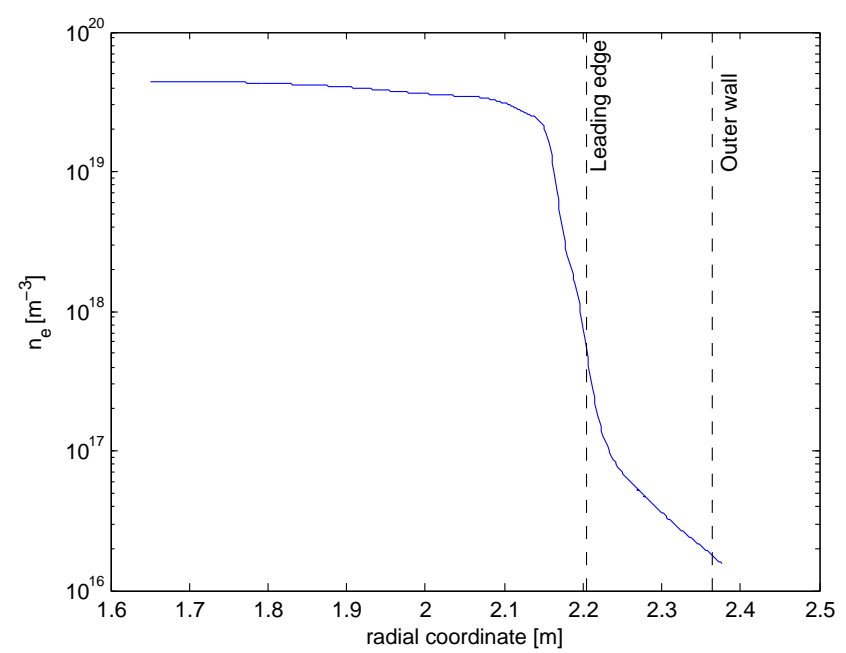

Figure 3. Density profile for ASDEX Upgrade experiment \#31278, used in our simulations.

the perpendicular DC conductivity (see section 5), are respectively $8 \cdot 10^{-4}$ and $4 \cdot 10^{-6}$. SSWICH-SW allows us to modify the density profile in the private SOL, but we have not done this: we use the same density profile inside the private SOL as outside it. The SSWICH-SW simulation domain extends, in the parallel direction, $0.5 \mathrm{~m}$ beyond the antenna limiters on both sides. The 2D SSWICH-SW geometry is discretized using a triangular mesh, with parameters as in table 1 . The mesh is scaled anisotropically: typical length scales of the triangular elements in the radial direction are $(\max \Delta) /$ (radial scaling), and similarly in the parallel direction.

\subsection{Phasing and power balance parameter scan}

The 3-strap antenna on ASDEX Upgrade was designed to minimize $E_{\|}$at the limiters, thereby (it is hoped) limiting the DC potential and associated ion sputtering [8]. The antenna has two ports, one which connects to the two outer straps, and one which connects to the central strap (figure 4). Linear electrodynamics [1] predicts that $E_{\|}$at the limiters is minimized if the antenna is run in dipole configuration (the input power on both ports is in phase), with about $70 \%$ of total power on the central strap and $30 \%$ on the side straps (so $15 \%$ each).

There is a difference in convention between $[1$, 8] and this paper: [1] defines the power balance as $P_{\text {center }} / P_{\text {outer }}$, and finds that the optimal power

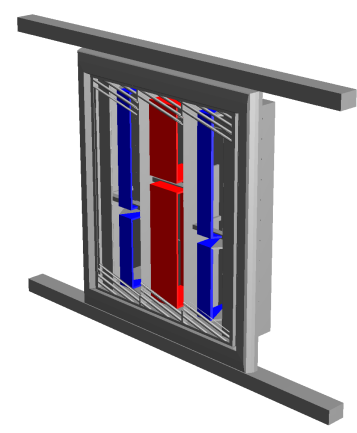

Figure 4. The RAPLICASOL model of the ASDEX Upgrade 3-strap antenna, with a part of the Faraday screen removed for clarity. The central strap is indicated in red, the outer straps in blue.

balance is around $P_{\text {center }} / P_{\text {outer }}=2$. Here, we define the power balance as $P_{\text {outer }} / P_{\text {total }}=P_{\text {outer }} /\left(P_{\text {outer }}+\right.$ $\left.P_{\text {center }}\right)$, and the corresponding optimal power balance is

$\frac{P_{\text {outer }}}{P_{\text {outer }}+P_{\text {center }}}=\frac{P_{\text {outer }}}{P_{\text {outer }}+2 P_{\text {outer }}}=\frac{1}{3}$

One might wonder if the linear analysis of [1] suffices: could nonlinear sheath effects be strong enough to shift the optimal operating parameters of the 3-strap antenna? We have used SSWICH-SW to calculate the DC potentials on the limiters of the 3strap antenna given the phasing and the power balance as inputs.

SSWICH-SW, in its default configuration, calculates the DC potentials at 6 toroidal locations: the inner and outer surfaces of both limiters (figure 1), as well as the outer sides of the simulation region. For each location, SSWICH-SW returns a 2D map of $V_{D C}$ vs. radial and poloidal coordinate (see figures 6 and 7 for example, and figure 5 for the coordinate system). It is the maximum of these $V_{D C}$ maps which is plotted in figure 8, at the locations of interest: the inner and outer surfaces of the limiters.

The parameters where the maximal DC potential is the lowest, where the least amount of ion sputtering is expected to occur, are phasing $=0^{\circ}$ (dipole configuration) and 30 to $40 \%$ of the power on the outer straps. This result agrees with what was expected from linear electrodynamics: the nonlinear sheath physics does not significantly modify the optimal antenna operating parameters.

Figure 8 is directly comparable with figure 9 , in which the maximal $E_{\|}$at the limiters is shown. This maximal $E_{\|}$was calculated with the finite-element code RAPLICASOL which we discussed in section 3 , and which does not take sheath boundary conditions into account. 


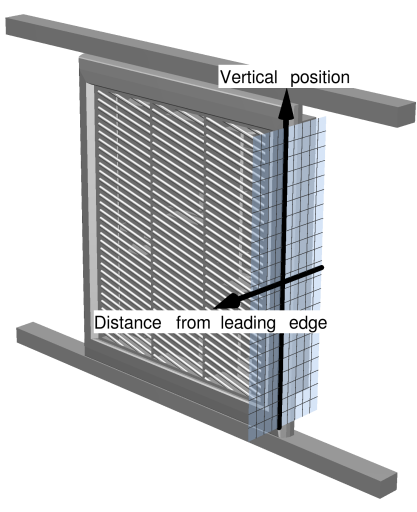

Figure 5. Clarification of the radial-vertical coordinate system used in figures $6,7,12,13,14$ and 15 . The "distance from leading edge" is the radial distance from the leading edge, positive towards the plasma and negative towards the outer wall.

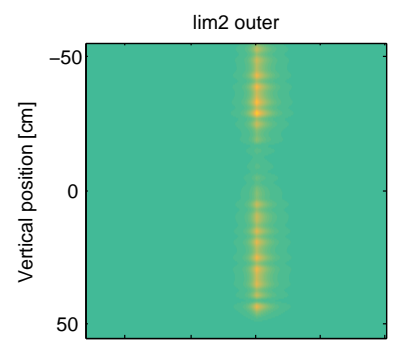

$\lim 1$ outer

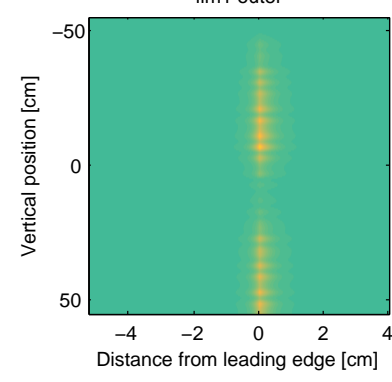

Distance from leading edge $[\mathrm{cm}]$

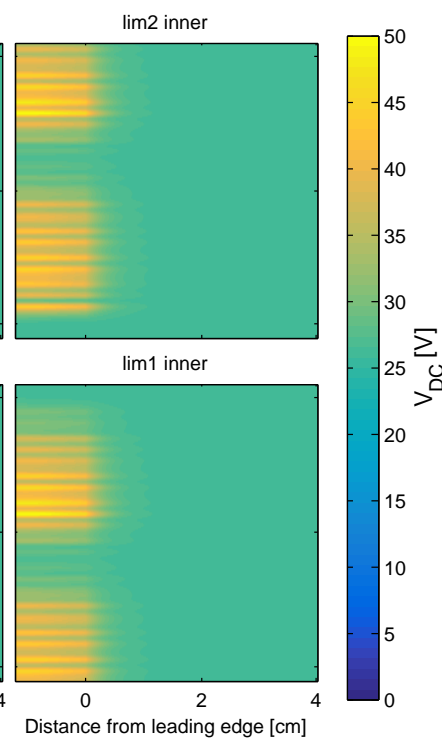

Figure 6. SSWICH-SW $V_{D C}$ field map on the inner and outer sides of both limiters for the optimized antenna operating parameters (power balance $P_{\text {center }} / P_{\text {total }}=0.4$, phasing $0^{\circ}$ ).

SSWICH-SW also returns field maps for the RF potential $V_{R F}$. We show the maximum $\left|V_{R F}\right|$ vs. the phasing and power balance in figure 10. Because of the way the fields in SSWICH-SW are excited $\left(E_{\|}\right.$ boundary condition in between the limiters, see section 2), $V_{R F}$ on the outer sides of the limiters is almost 0 , because it only gets excited by the evanescent slow wave travelling around the limiters. We deem this nonphysical and choose to only show $V_{R F}$ on the inner sides in figure 10.

$V_{D C}$, on the other hand, differs significantly from the floating potential even on the outer sides of the limiters, because of the relative ease with which currents $J_{D C}=-\left(\sigma_{\|} \nabla_{\|}+\sigma_{\perp} \nabla_{\perp}\right) V_{D C}$ from the private SOL can connect to the outer sides of the limiters.

From (4) and (5), we see that $V_{D C}$ on a given
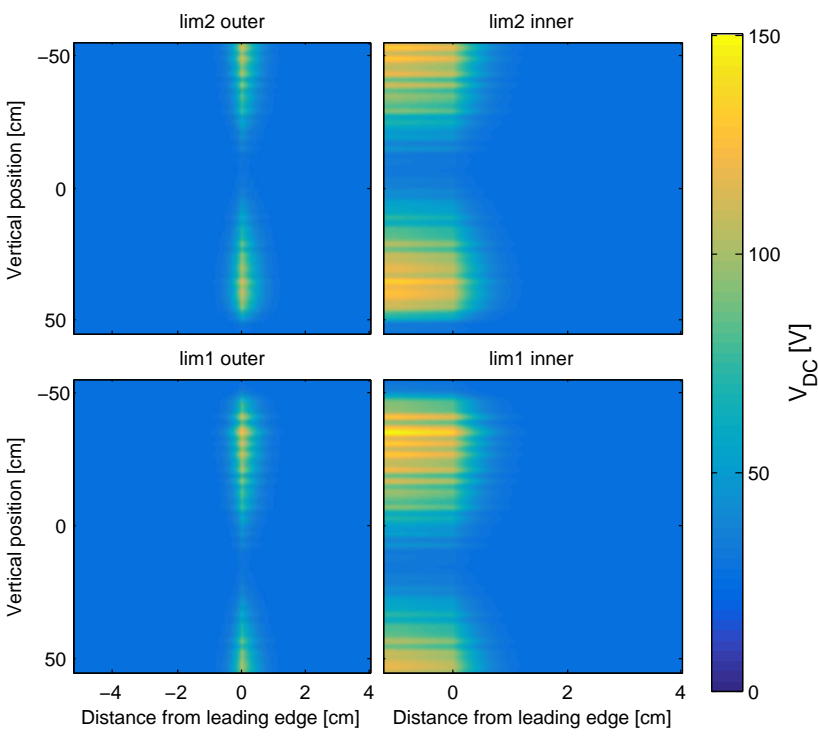

Figure 7. SSWICH-SW $V_{D C}$ field map on the inner and outer sides of both limiters for unoptimized antenna operating parameters (power balance $P_{\text {center }} / P_{\text {total }}=0.0$, phasing $36^{\circ}$ ).

open field line depends on $V_{R F}$ at the two field line extremities, as well as on $V_{D C}$ on the surrounding field lines. From this, we can guess that if $\left|V_{R F}\right|$ is reduced over all significant sheath boundaries, then the (nonlinear) $V_{D C}$ will also be reduced everywhere in the simulation domain. Indeed this is what we see: the global minimum in figure 8 occurs at the same parameters as in figure 10 .

\subsection{Dipole case}

4.3.1. Power balance parameter scan Even the fairly coarse parameter scan of the previous subsection consists of 110 SSWICH-SW simulations and took about a month to complete on a computer with 2 8-core $3.40 \mathrm{GHz}$ processors. For the dipole case, we have refined the grid using power balance steps of 0.05 instead of 0.1 .

In figure 11, we show the vertically-averaged radial maximum of the RF and $\mathrm{DC}$ potential from SSWICH-SW, as well as the vertically-averaged $\left|E_{\|}\right|$ from RAPLICASOL, vs. power balance for the dipole case. The vertical average is taken over a $10 \mathrm{~cm}$ vertical range at the top and at the bottom of the antenna.

The relation between $E_{\|}$and $V_{R F}$ is of particular interest in view of discussion in paper [1], where calculated values of $E_{\|}$were used to compare with local experimental RF amplitude measurements at antenna limiters. The measurements relate closer to $V_{R F}$ rather than to $E_{\|}$. In figure 11 we see that $E_{\|}$is not systematically more sensitive to the power balance than $V_{R F}$, and the precise relationship between them is location-dependent. 


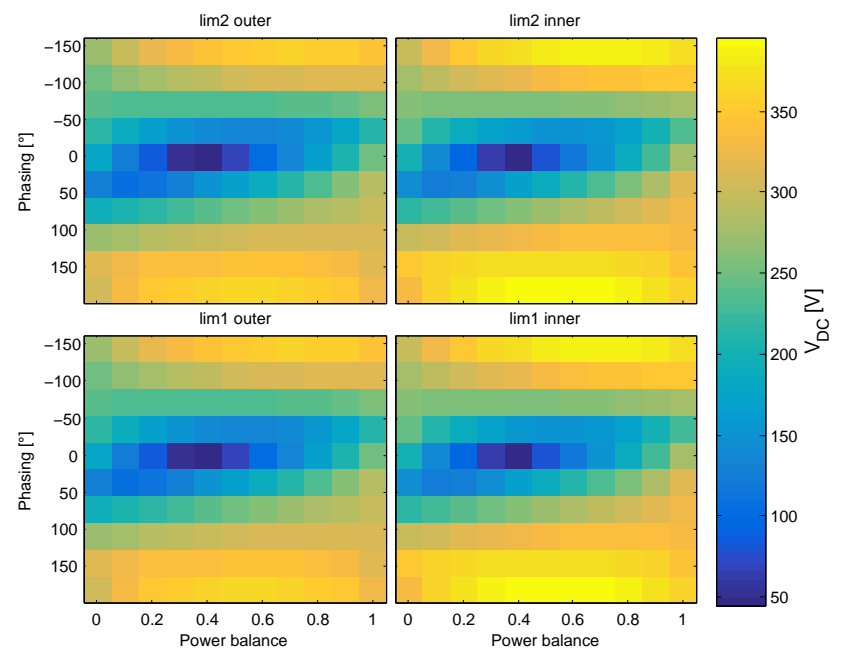

Figure 8. Numerically calculated maximal DC potential on the limiter vs. antenna power balance and phasing.
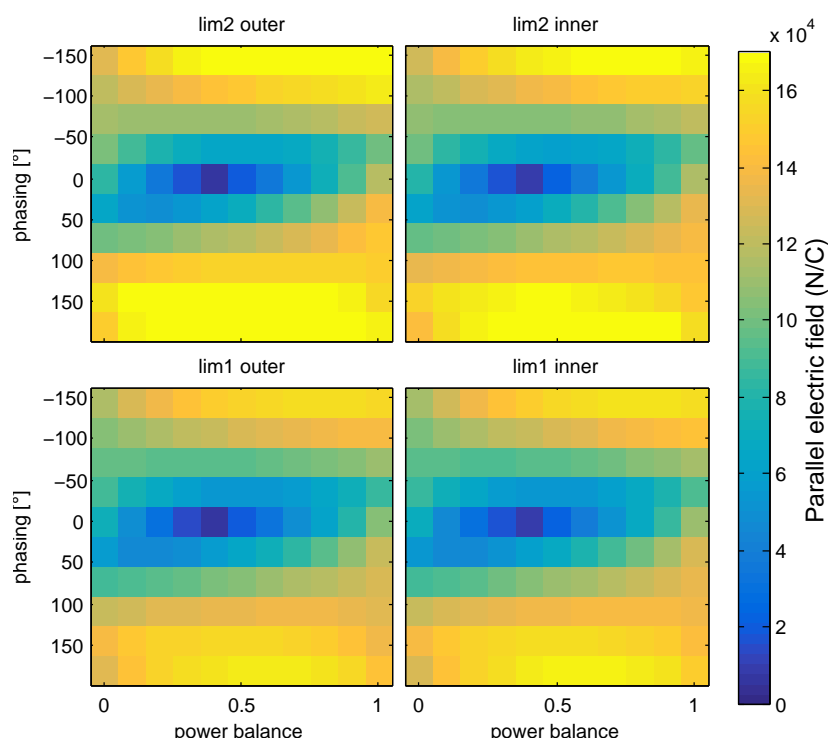

Figure 9. Numerically calculated maximal $E_{\|}$at the sides of the limiters, as calculated with the linear finite-element code RAPLICASOL, which does not take sheath physics into account (it imposes only PEC boundary conditions on metallic elements).

4.3.2. Minimizing $V_{D C}$ locally Still in the dipole case, for every coordinate in the simulation region, we can ask the questions

(i) By how much can we change $V_{D C}$ at this particular coordinate by changing the power balance? The difference between the maximal achievable $V_{D C}$ and the minimal achievable $V_{D C}$ is shown in figure 12.

(ii) which power balance would minimize $V_{D C}$ at this particular coordinate? The answer is shown in figure 13 .

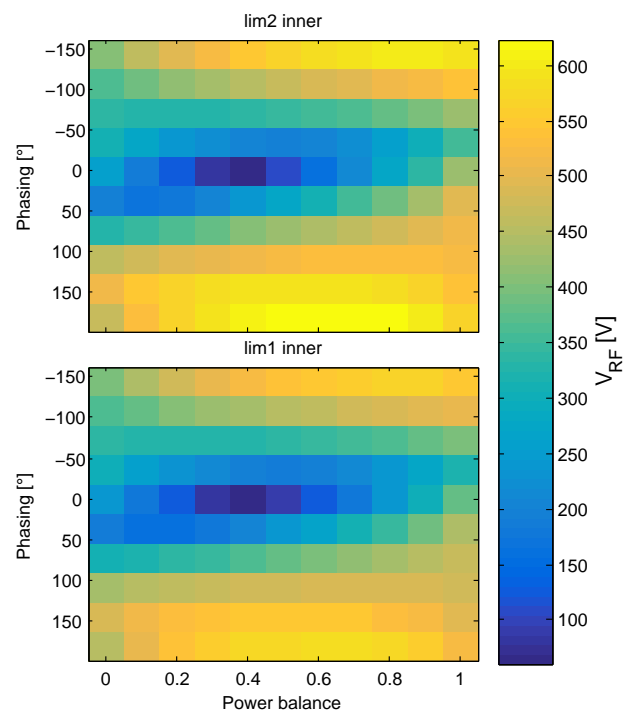

Figure 10. Numerically calculated maximal RF potential on the inner sides of the limiters vs. antenna power balance and phasing.

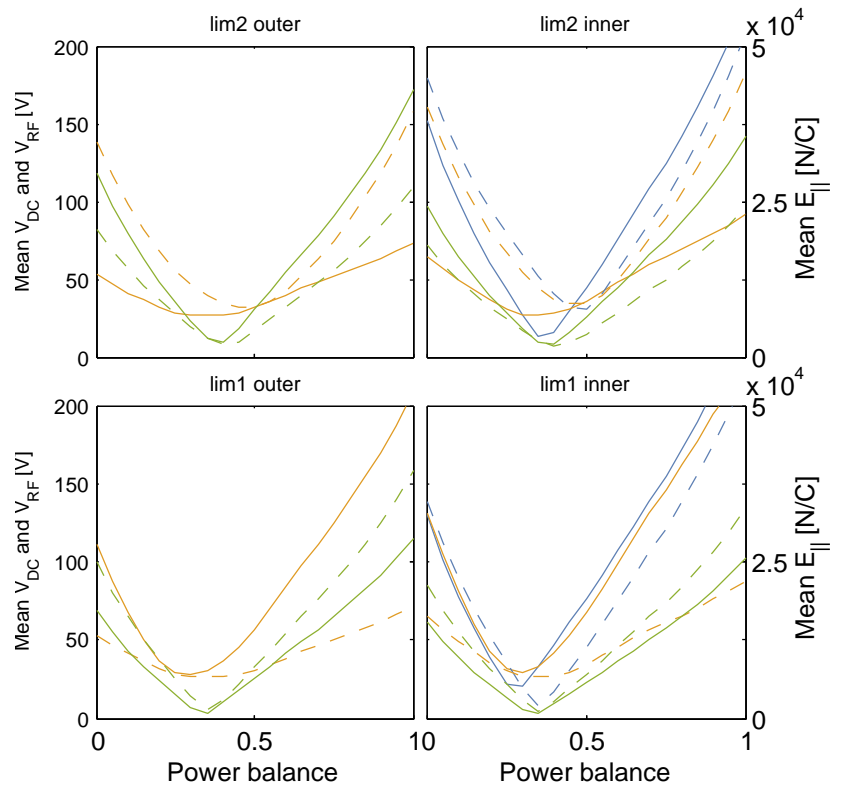

Figure 11. Numerically calculated vertically-averaged radial maximum of $V_{R F}$ and $V_{D C}$, and vertically-averaged $E_{\|}$for the dipole case (phasing is $0^{\circ}$ ) vs. antenna power balance. The average is taken over a $10 \mathrm{~cm}$ vertical region at the top (solid lines) and bottom (dashes lines) of the antenna. Green: $E_{\|}$, orange: $V_{D C}$, blue: $V_{R F}$ (not shown on the outer sides). 


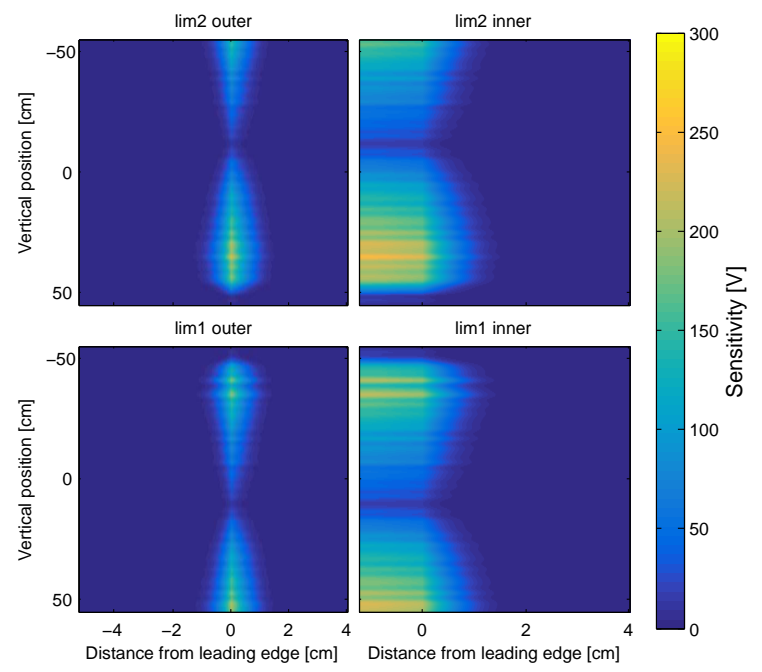

Figure 12. Sensitivity of the local $V_{D C}$ to changes in the power balance, for the inner and outer sides of the left and right limiter.

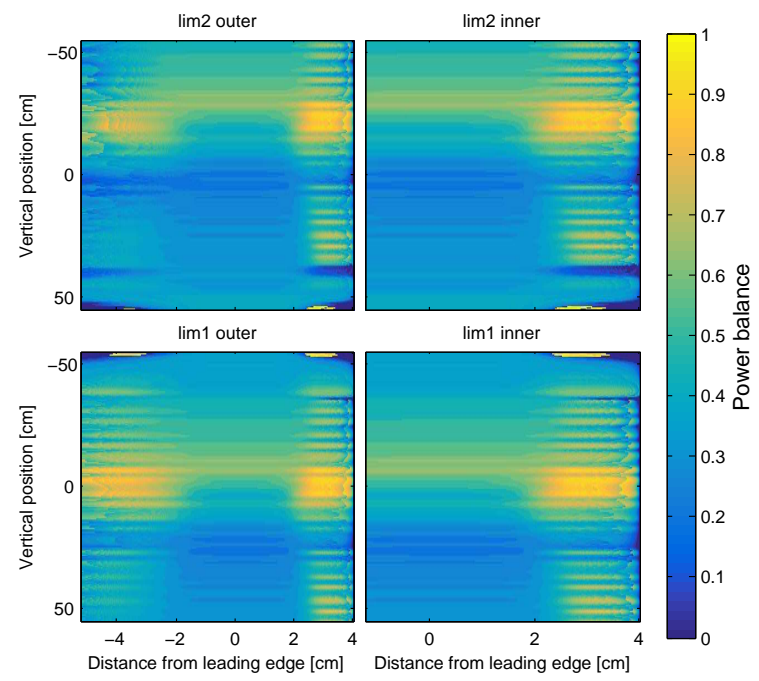

Figure 13. Power balance which minimizes the local $V_{D C}$, for the inner and outer sides of the left and right limiter.

\subsection{Minimizing $V_{R F}$ locally}

For $V_{R F}$, we can ask the same questions as for $V_{D C}$ in section 4.3.2. This case is somewhat simpler, because in the approximations made by SSWICH-SW in its asymptotic mode, $V_{R F}$ is a linear function of the port voltages $V_{1}$ and $V_{2}$ :

$$
V_{R F}(\vec{r})=V_{R F, 1}(\vec{r}) V_{1}+V_{R F, 2}(\vec{r}) V_{2}
$$

where the per-port responses $V_{R F, 1}(\vec{r})$ and $V_{R F, 2}(\vec{r})$ can be obtained from SSWICH-SW. This lets us calculate which $V_{1} / V_{2}$ will make $V_{R F}$ locally 0 :

$$
\begin{aligned}
& 0=V_{R F, 1}(\vec{r}) V_{1}+V_{R F, 2}(\vec{r}) V_{2} \\
& \frac{V_{1}}{V_{2}}=-\frac{V_{R F, 2}(\vec{r})}{V_{R F, 1}(\vec{r})}
\end{aligned}
$$

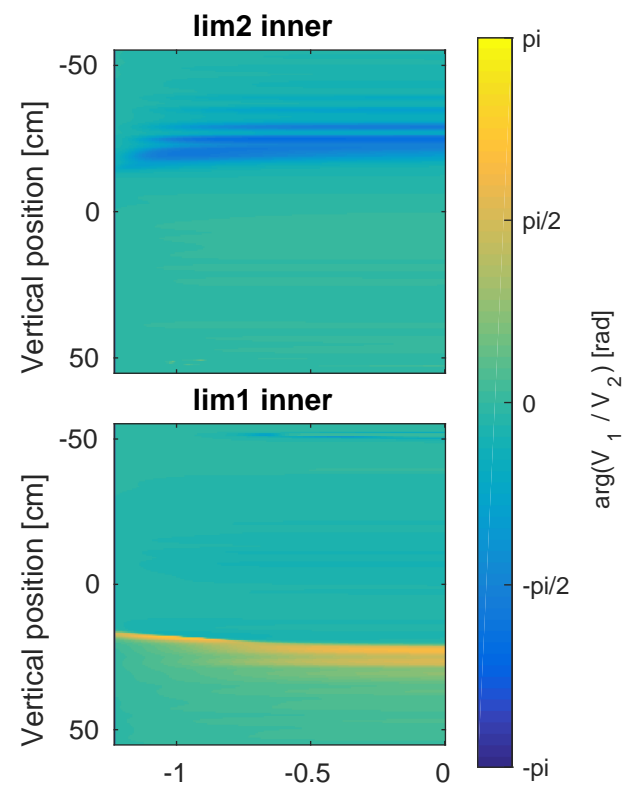

Distance from leading edge $[\mathrm{cm}]$

Figure 14. Argument of the ratio of port voltages which minimizes the local $\left|V_{R F}\right|$, on the inside of both limiters. ( $V_{R F}$ returned by SSWICH-SW on the outside of the limiters is near zero in any case)

Naturally this optimal $\frac{V_{1}}{V_{2}}$ is a complex quantity. In figures 14 and 15, we plot its argument and absolute value on the inner sides of the limiters, where $V_{R F}$ is meaningfully calculated by SSWICH-SW.

As expected, the argument of the optimal $\frac{V_{1}}{V_{2}}$ is close to 0 almost everywhere: $V_{R F}$ is minimized when the antenna operates in dipole configuration. The optimal $\frac{V_{1}}{V_{2}}$ is not constant: $V_{R F}$ cannot be made zero everywhere. Only overall reduction is achievable, and only for good antenna designs.

\section{Sensitivity to SSWICH-SW transport parameters}

As we mentioned in section 4.1, version 10 of the SSWICH-SW code uses two transport parameters $R_{s, f p}$ and $R_{s, p p}$ to phenomenologically model the perpendicular DC conductivity. The perpendicular conductivity $\sigma_{\perp}$ in the private scrape-off layer $\left(\sigma_{\perp, p}\right)$ and in the free scrape-off layer $\left(\sigma_{\perp, f}\right)$ are assumed to be proportional to the parallel (Spitzer) conductivity $\sigma_{\|}$as follows:

$\begin{aligned} \sigma_{\perp, p} & \equiv R_{s, p p} \sigma_{\|} \\ \sigma_{\perp, f} & \equiv R_{s, f p} \sigma_{\|}\end{aligned}$

These values are not directly constrained by experiment. We have chosen them such that the radial extent of the $V_{D C}$ patterns (figures 6 and 7 ) approximately matches experimental observation. 


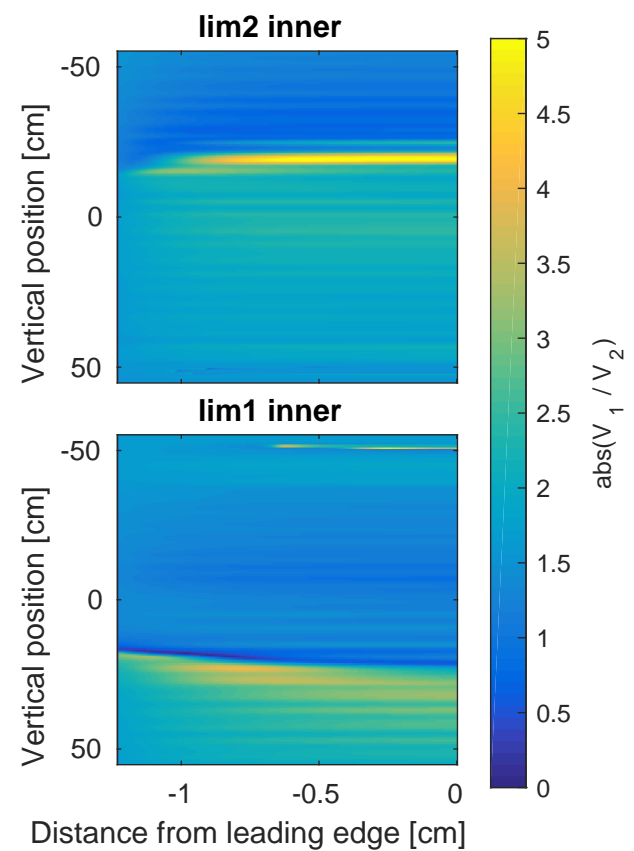

Figure 15. Absolute value of the ratio of port voltages which minimizes the local $\left|V_{R F}\right|$, on the inside of both limiters. ( $V_{R F}$ returned by SSWICH-SW on the outside of the limiters is near zero in any case)

We believe that at these values of the transport parameters, the sheath in the private SOL is saturated: as much current is drawn from it as possible. Thus, the magnitude of $V_{D C}$ at the limiter edge should not be much influenced by small changes in these parameters. Figure 16 confirms this. $V_{D C}$ at the limiter edge barely changes when we change $R_{s, p p}$ between $4 \cdot 10^{-6}$ and $8 \cdot 10^{-6}$. For lower values of $R_{s, p p}$, the sheath is no longer saturated and $V_{D C}$ decreases.

\section{Conclusion}

We have used the sheath-modeling code SSWICHSW in its asymptotic mode (suppressing the nonlinear dependence of the sheath width on $V_{D C}$ ) to calculate the influence of the operating parameters of the ASDEX Upgrade 3-strap antenna (phasing and power balance) on the DC potential at the antenna limiters, a quantity which we wish to minimize in order to avoid ion sputtering. Our results agree with what was expected from prior computations using only linear physics: the nonlinear sheath physics does not significantly alter the optimal antenna operating parameters. Furthermore, for the plasma and geometrical parameters considered in this paper, $E_{\|}$calculated by RF solvers can indeed be used as a proxy for the optimization as in [1].

Unfortunately, there are no antenna operating parameters which cancel either $V_{R F}$ or $V_{D C}$ everywhere:

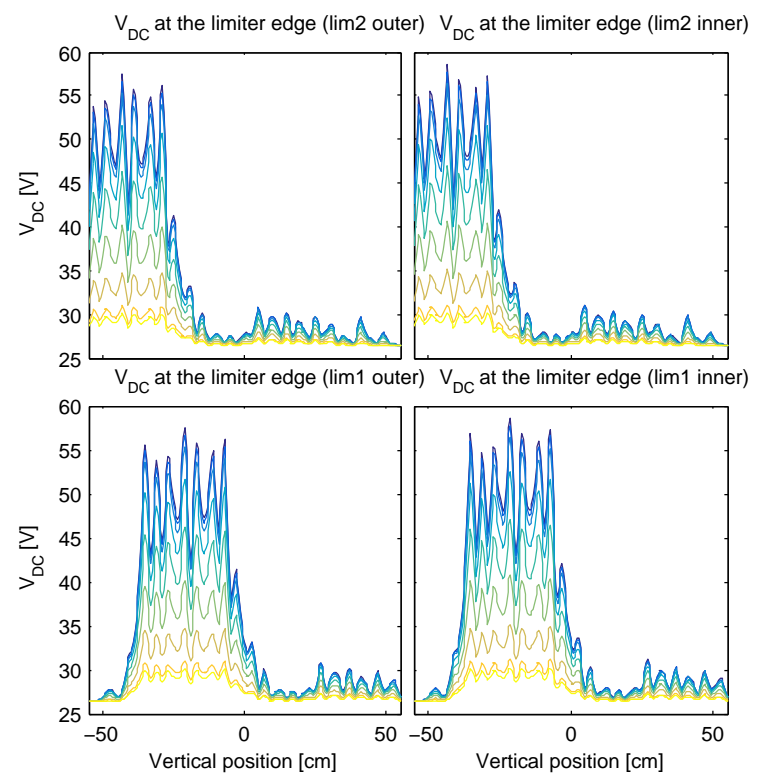

Figure 16. Sensitivity of $V_{D C}$ to variations in the SSWICHSW transport parameter $R_{s, p p}$ from $8 \cdot 10^{-7}$ (yellow) through $8 \cdot 10^{-6}$ (dark blue).

only local cancellation is possible. The particular structure of the 3-strap antenna also allows overall partial reduction of $\left|V_{R F}\right|$, which ensures also overall $V_{D C}$ reduction.

We discussed two linear "indicators" of the nonlinear $V_{D C}: E_{\|}$and $V_{R F}$ (the latter is linear only within the approximations made in SSWICH-SW's asymptotic mode). In our numerical simulations we find that overall reduction of the two indicators is obtained for the same electrical settings, close to the optimal settings found experimentally. This similar behaviour of $V_{R F}$ and $E_{\|}$could be explained as follows:

- Both RAPLICASOL and SSWICH-SW propagate the Slow Wave in the plasma from a map of $E_{\|}$ existing at the antenna mouth. RAPLICASOL produces this map self-consistently from the RF voltages imposed on the antenna structure.

- RAPLICASOL and SSWICH-SW differ in the boundary conditions applied on plasma-touching boundaries, as well as the presence of full polarization in RAPLICASOL. $V_{R F}$ is related to the parallel derivative of $E_{\|}$at the sheath boundaries via the radial diffusion equation (3).

- In asymptotic mode of SSWICH-SW, Slow Wave evanescence produces toroidal proximity effects in RF sheath excitation: [6] clearly demonstrated that $V_{R F}$ amplitude at the antenna limiters mainly depends on the $E_{\|}$amplitudes at the toroidal extremities of the applied field map.

- If we assume that $E_{\|}$fields in RAPLICASOL mainly come from the Slow Wave, then similar 
proximity effects could be invoked to argue that the $E_{\|}$on the side limiters (without sheath boundary conditions) also depends mainly on the $E_{\|}$at the toroidal extremities of the field map.

From this, we might tentatively conclude that the two linear indicators $V_{R F}$ and $E_{\|}$are both sensitive to toroidal proximity effects, and likely behave in a similar way.

This work has been carried out within the framework of the EUROfusion Consortium and has received funding from the Euratom research and training programme 2014-2018 under grant agreement No 633053. The views and opinions expressed herein do not necessarily reflect those of the European Commission.

\section{References}

[1] V Bobkov, D Aguiam, R Bilato, S Brezinsek, L Colas, $\mathrm{H}$ Faugel, H Fünfgelder, A Herrmann, J Jacquot, A Kallenbach, et al. Making ICRF power compatible with a high-Z wall in ASDEX Upgrade. Plasma Physics and Controlled Fusion, 59(1):014022, 2016.

[2] V. Bobkov, M. Balden, R. Bilato, F. Braun, R. Dux, A. Herrmann, H. Faugel, H. Fünfgelder, L. Giannone, A. Kallenbach, H. Maier, H.W. Müller, R. Neu, J.M. Noterdaeme, Th. Pütterich, V. Rohde, N. Tsujii, F. Zeus, H. Zohm, and the ASDEX Upgrade Team. ICRF operation with improved antennas in ASDEX upgrade with W wall. Nuclear Fusion, 53(9):093018, 2013.

[3] J. Jacquot. Description non-linéaire auto-cohérente de la propagation d'ondes radiofréquences et de la périphérie d'un plasma magnétisé. $\mathrm{PhD}$ thesis, Université de Lorraine, 2013.

[4] J. Jacquot, D. Milanesio, L. Colas, Y. Corre, M. Goniche, J. Gunn, S. Heuraux, and M. Kubič. Radio-frequency sheaths physics: Experimental characterization on tore supra and related self-consistent modeling. Physics of Plasmas, 21(6):061509, 2014.

[5] L. Colas, J. Jacquot, S. Heuraux, E. Faudot, K. Crombé, V. Kyrytsya, J. Hillairet, and M. Goniche. Self consistent radio-frequency wave propagation and peripheral direct current plasma biasing: Simplified three dimensional nonlinear treatment in the "wide sheath" asymptotic regime. Physics of Plasmas, 19(9):092505, 2012.

[6] L. Colas, L. Lu, A. Křivská, J. Jacquot, J. Hillairet, W. Helou, M. Goniche, S. Heuraux, and E. Faudot Spatial proximity effects on the excitation of sheath RF voltages by evanescent slow waves in the ion cyclotron range of frequencies. Plasma Physics and Controlled Fusion, 59(2):025014, 2017.

[7] L. Lu. Modelling of plasma-antenna coupling and nonlinear radio frequency wave-plasma-wall interactions in the magnetized plasma device under ion cyclotron range of frequencies. PhD thesis, Ghent University, 2016.

[8] V Bobkov, F Braun, R Dux, A Herrmann, H Faugel, H Fünfgelder, A Kallenbach, R Neu, J-M Noterdaeme, $\mathrm{R}$ Ochoukov, et al. First results with 3-strap ICRF antennas in ASDEX Upgrade. Nuclear Fusion, 56(8):084001, 2016. 